\title{
Dancing in a Roar: Multilobed Aneurysm Arising From Azygous Anterior Cerebral Artery
}

\section{Karki $\mathrm{KT}^{1 *}$ and Karki B ${ }^{2}$}

${ }^{1}$ Department of Neurosurgery, College of Medical Sciences, Bharatpur, Chitwan, Nepal

${ }^{2}$ Department of Radiology, PT Birtacity Hospital, Birtamode, Jhapa, Nepal

*Corresponding author: Karki KT, Department of Neurosurgery, College of Medical Sciences, Bharatpur, Chitwan, Nepal, Tel: 977-9843405124; E-mail: tamrakarkaruna@gmail.com

Rec: July 22, 2016, Acc: February 20, 2017, Pub: February 28, 2017

Copyright: (c) 2017 Karki KT, et al. This is an open-access article distributed under the terms of the Creative Commons Attribution License, which permits unrestricted use, distribution, and reproduction in any medium, provided the original author and source are credited.

\section{Short Communication}

Embryologically, anterior cerebral artery (ACA) takes a medial course in 40-day period. Anterior communicating artery develops after 4 days. Along with the development of corpus callosum, ACAs course in a frontal caudal direction [1]. ACA arises from the internal carotid artery distal to the carotid siphon, crosses the anterior perforating substance. It gives off small medial striate vessels and reaches the interhemispheric fissure and connects with the contralateral ACA by the anterior communicating artery. It then turns upward in the direction of the genu of the corpus callosum with which it is intimately associated as it parallels it in a wide arc to continue over the medial surface of the hemisphere [1]. It may occasionally lie in the cingulate sulcus. Right and left ACA vessels run in parallel. Left may possibly course slightly posterior than the right one. It supplies orbitofrontal and medial hemispheric portions of the human brain. The unusual fusion of the paired A2 is either originates from the medial branch of the olfactory artery at the initial $16 \mathrm{~mm}$ stage during embryonic development or the continuation of the median artery in the corpus callosum at the 24-mm stage. [2] Among the lower primates, there is no anterior communicating artery. Unpaired ACA is normal in snakes, tortoises, and crocodiles [3]. However, it is a rare variation in human in which A2 segments of both ACAs are represented by a single common vessel. It may have profound clinical implications with an incidence of 1.15 to $5 \%[1,2,4]$ which is either due to lack of development or regression of anterior cerebral artery during embryogenesis. Different types of azygos ACA has been described in the literature [4].

Type I is a classical type. All the major vessels arise from the single stump feed most of both anterior cerebral hemispheres as well as the corpus callosum (Figure 1). This is usually seen in the anterior median fissure supplying to both hemispheres. Type II has a short median stem of azygos ACA. This divides into further branches to supply both the hemispheres separately. Type III represents two separate A2 segments. Type IV shows an azygos pericallosal artery. Anterior cerebral circulation appears normal except for the pericallosal vessels. Type $\mathrm{V}$ has a third azygos median A2 artery. There are several variations of cerebral vasculatures. Hypoplasia, aplasia and duplications of the precommunicating segment of ACA, duplication, and fenestration of anterior communicating artery, azygos ACA, and variation in an A2 segment of ACA in its terminal branches are among them [4]. The incidence of an aneurysm ranges from $13 \%$ to $71 \%$ in such kind of variation due to altered hemodynamics and defect in tunica media promoting the aneurysmal growth and rupture [5]. Another reason for the frequent existence of an aneurysm in the ACA is being the most primitive vessel in Circle of Willis. Arteriovenous malformation, agenesis of corpus callosum, bilateral ischemia, holoprosencephaly, and hydranencephaly are other frequent intracranial abnormalities associated with primitive ACA [1-5].

Variation in anterior circulation poses a great surgical importance. Although fenestration, duplication or triplication has a protective mechanism for any kind of occlusion or vasospasm, however, the azygous vessel is more prone to produce grave consequences during the endovascular or surgical intervention. Although Circle of Willis has the ability for potential shunting effect during vascular compression, surgical intervention is more challenging since it may possess more risk of ischemia of both frontal lobes if the narrowing is produced. Azygous ACA with a multilobed aneurysm in Author's CT angiographic image (Figure 1) represents an archetypical picture of performing an art of ballet dance. Azygous ACA with an aneurysm pointing upward corresponds as a ballistic move. Both of her arms and the entire body has been bent backward in an arc like fashion in a single breath. Such move is made in a critical instance. Similarly, the azygous ACA carrying multiple aneurysms and feeding both anterior cerebral hemispheres in given image stands in the most life threatening condition. Therefore, clinical significance of solitary ACA is very important to consider while clipping or coiling.

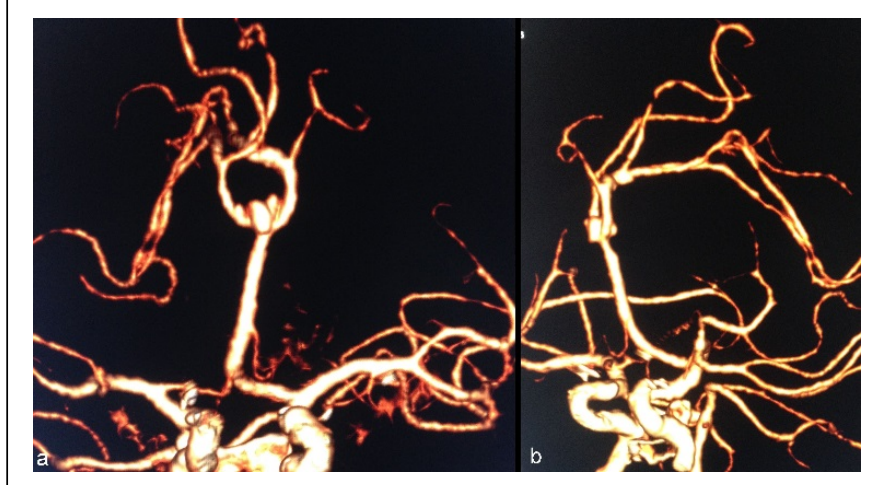

Figure 1: Major vessels arise from the single stump feed most of both anterior cerebral hemispheres as well as the corpus callosum.

\section{References}

1. Binatl O, Ozer FD, Aydin M, Cicek E (2013) Azygos anterior cerebral artery aneurysm with concomittant vascular anomaly: A case report. World J Neurosci 3: 9-51. 
Citation: Karki TK, Karki B (2017) Dancing in a Roar: Multilobed Aneurysm Arising From Azygous Anterior Cerebral Artery. J Neurol Disord 5: 331. doi:10.4172/2329-6895.1000331

Page 2 of 2

2. Stefani MA, Schneider FL, Marrone AC, Severino AG, Jackowski AP, et al (2000) Anatomic variations of anterior cerebral artery cortical branches. Clin Anat 13: 231-236.

3. LeMay M, Gooding CA (1966) The clinical significance of the azygos anterior cerebral artery (A.C.A.). Am J Roentgenol Radium Ther Nucl Med 98: 602-610.
4. Gunnal SA, Wabale RN, Farooqui MS (2013) Variations of anterior cerebral artery in human cadavers. Neurology Asia 18: 249-259.

5. Huh JS, Park SK, Shin JJ, Kim TH (2007) Saccular aneurysm of the azygos anterior cerebral artery: Three case reports. J Korean Neurosurg Soc 42: 342-345. 